\title{
The radial velocity curve of HD 153919 (4U 1700-37) revisited ${ }^{\star}$
}

\author{
G. Hammerschlag-Hensberge ${ }^{1}$, M. H. van Kerkwijk ${ }^{2,3}$, and L. Kaper ${ }^{1}$ \\ 1 Astronomical Institute “Anton Pannekoek” and Center for High-Energy Astrophysics, University of Amsterdam, \\ Kruislaan 403, 1098 SJ Amsterdam, The Netherlands \\ 2 Astronomical Institute, Utrecht University, PO Box 80000, 3508 TA Utrecht, The Netherlands \\ 3 Dept. of Astronomy \& Astrophysics, 60 St George Street, Toronto, ON, M5S 3H8, Canada
}

Received 25 March 2003 / Accepted 17 June 2003

\begin{abstract}
We have re-analysed all available high-resolution ultraviolet IUE spectra of the high-mass X-ray binary HD 153919/4U 1700-37. The radial velocity semi-amplitude of $20.6 \pm 1.0 \mathrm{~km} \mathrm{~s}^{-1}$ and orbital eccentricity of $0.22 \pm 0.04$ agree very well with the values obtained earlier from optical spectra. They disagree with earlier conclusions for the same data reduced by Heap \& Corcoran (1992) and by Stickland \& Lloyd (1993).
\end{abstract}

Key words. stars: binaries: close - stars: early type - stars: radial velocities - stars: individual: HD 153919 -

4U 1700-37 - ultraviolet: stars

\section{Introduction}

The bright Of star HD 153919 is the optical counterpart of the 3.4 day eclipsing high-mass X-ray binary 4U 1700-37, discovered by Jones et al. (1973). About 2.5 million years ago, the binary system escaped from the OB association Sco OB2 after the supernova explosion that formed the compact companion (Ankay et al. 2001). The X-ray source powered by the stellar wind of the Of star shows no regular pulsations and the highly variable X-ray flux is less than expected from standard wind accretion (e.g. Rubin et al. 1996; Kaper 1998). The decrease in orbital period determined from mid-eclipse observations and first mentioned by Haberl et al. (1989) was confirmed by Rubin et al. (1996) and is probably due to wind-driven angular momentum loss from the Of star. Because no direct measurements can be made of the orbital motion of the compact object, system parameters depend heavily on radial velocity studies of the optical companion. The most recent analysis of the physical parameters cannot resolve the nature of the compact object in this system (Clark et al. 2002). Its likely mass is considerably lower than those found for black holes, but it is significantly in excess to fit the high density nuclear equation of state for neutron stars. A point to remember, however, is that also the Vela X-1 pulsar has a high mass, comparable to that of 4U 1700-37 (van Kerkwijk et al. 1995).

Send offprint requests to: G. Hammerschlag-Hensberge, e-mail: godeliev@science.uva.nl

* Based on observations obtained with the International Ultraviolet Explorer.
The most thorough study of radial velocities of lines in the visible spectral region has been made by Hammerschlag-Hensberge (1978). It is clear from the observations that the strongly variable stellar wind in this system complicates a straightforward determination of the system parameters. The results for the orbital analysis of all lines together in the visible blue spectral region (3700-4900 $\AA$ ) yield a semi-amplitude $K=19 \pm 1 \mathrm{~km} \mathrm{~s}^{-1}$ and an orbital eccentricity $e=0.16 \pm 0.08$.

Heap \& Corcoran (1992) have examined the system parameters using the available ultraviolet spectra of HD 153919. They find $K=18 \pm 3 \mathrm{~km} \mathrm{~s}^{-1}$ and a negligible eccentricity. For their investigation they cross-correlated a few selected narrow regions of the ultraviolet spectra with the same regions of one of the spectra. To bring their spectra to the same frame of reference they used the heliocentric correction. However, instrumental and processing shifts during the reduction of spectra obtained with the International Ultraviolet Explorer (IUE) require a more sophisticated approach (Stickland 1992).

Stickland \& Lloyd (1993) re-examined the same data, now cross-correlating both stellar and interstellar spectra with reference to one of the UV spectra. They used the complete ultraviolet spectral range excluding P-Cygni lines whose profiles are affected by mass loss. Amazingly, they found a much smaller semi-amplitude $K$ of $10 \mathrm{~km} \mathrm{~s}^{-1}$ and an eccentricity of 0.17 .

In a similar study of the high-mass X-ray binary HD 77581/Vela X-1 Barziv et al. (2001) found a disagreement in the semi-amplitude of the radial velocity curve with the results of Stickland et al. (1997) for this system. This strengthened our decision to re-analyse the ultraviolet spectra 
of HD 153919 again. In Sect. 2 we describe the observations and the data reduction procedures. In Sect. 3 we present the resulting radial velocity curve and in Sect. 4 we compare our results with the ones found by Heap \& Corcoran and by Stickland $\&$ Lloyd. In Sect. 5 we summarize our conclusions and discuss the evolutionary consequences.

\section{Observations and data reduction}

A large number of ultraviolet spectra of HD 153919 have been obtained with the International Ultraviolet Explorer. The spectra obtained with the Short Wavelength Prime Camera in highresolution mode are listed in Table 1. A separate analysis of the same IUE spectra was performed by Stickland \& Lloyd (1993). These authors cross-correlated the set of spectra with one particular spectrum (SWP25600). Because their results were in large discrepancy with results from optical data we decided to re-analyse the same IUE data. We cross-correlated each spectrum with all other spectra of the data set to reduce errors. We did not use a template spectrum of another star with identical spectral type as independent reference spectrum - this is not realistic for such hot mass-losing stars which each have unique spectra - nor did we use an average spectrum to avoid autocorrelation effects leading to a systematic decrease of the amplitude of the radial velocity curve.

First the spectra - initially mapped onto a uniform wavelength grid of $0.05 \AA$ - were transformed to a logarithmic wavelength scale such that the Doppler shift becomes a linear displacement along the spectrum. The relevant part of the cross-correlation function is the area close to the top; it is this upper part which is fitted with an analytic function (a Gaussian plus a linear function) whose maximum determines the velocity shift of the spectrum. Because the cross-correlation profiles of IUE spectra show small excess peaks (see Evans 1988 and Fig. 4 in Van Kerkwijk et al. 1995) due to the so-called fixed-pattern noise in the detector, we corrected for those by including an extra Gaussian peak in the fit. For details on the cross-correlation method and instructive figures we refer to Van Kerkwijk (1993).

The spectral regions which were used for the crosscorrelation are listed in Table 2. The regions were chosen in a way to exclude parts of the spectrum containing variable line profiles such as the well-known P-Cygni profiles (see for instance Hammerschlag-Hensberge et al. 1990); also the spectral region including variable emission lines 1540-1740 ̊ (Kaper et al. 1990) was excluded. Table 2 lists the wavelength regions including interstellar lines which were used to place the individual spectra onto the same frame of reference, because IUE spectra have a variety of instrumental and processing shifts, as mentioned before.

\section{Results}

In Table 1 we list the radial velocities derived from the crosscorrelations of all spectra with each other. In Fig. 1, we show the radial velocities we obtained from the IUE spectra as a function of orbital phase. Because the spectra were cross-correlated with each other, no $\gamma$-velocity has been determined for the system and the velocities in the figure have been shifted to zero system velocity. The $\gamma$-velocity has been determined elsewhere (e.g. Gies \& Bolton 1986; Humphreys 1978): $-60 \mathrm{~km} \mathrm{~s}^{-1}$. It was not included in our figure because the value - which is rather uncertain - did not follow from our data. At first glance, it is already clear that the data show significant deviations from a smooth Keplerian curve. We believe these are intrinsic to the star, since earlier optical observations have already shown that the star has intrinsic variations (e.g., Kaper et al. 1994). As we will find that the deviations are substantially larger than the measurement errors, we give each measurement equal weight in our fits below.

We first fit a circular orbit, shown by the long-dashed line in Fig. 1, and find $K=18.7 \pm 1.0 \mathrm{~km} \mathrm{~s}^{-1}$. The root-mean-square (rms) residual is $6.36 \mathrm{~km} \mathrm{~s}^{-1}$. This is a factor two larger than the mean error in the measured velocities (using the formal errors, one finds a reduced $\chi^{2}=3.6$ ).

The residuals relative to the best-fit circular orbit are shown in the lower panel of Fig. 1. These seem to show a doublewave pattern, suggestive of an eccentric orbit. Fitting an eccentric orbit, we find eccentricity $e=0.22 \pm 0.04$, periastron angle $\omega=49^{\circ} \pm 11^{\circ}$, and the radial-velocity amplitude is $K=20.6 \pm 1.0 \mathrm{~km} \mathrm{~s}^{-1}$. The rms residuals are slightly lower, at $5.85 \mathrm{~km} \mathrm{~s}^{-1}$. Doing an F-test, this is not a significant improvement. The F-test, however, ignores the correlations in the residuals. In order to obtain a more robust estimate of the significance, we ran Monte-Carlo simulations, in which we made artificial data sets in which the velocity at each measurement phase was given by the sum of the velocity expected from the best-fit circular orbit and a random deviation. For the latter, we used two components, one to mimic the measurement error (i.e., a random number drawn from a normal distribution with standard deviation equal to the measurement error), and one to mimic the intrinsic deviations. For the intrinsic deviations, we again assumed a normal distribution, with a standard deviation of $6 \mathrm{~km} \mathrm{~s}^{-1}$, so that our artificial data sets have the same rms residuals as our data. Our simulations confirmed our error estimate on the eccentricity, and showed that the probability of obtaining an eccentricity of 0.22 by chance due to the deviations was negligible.

We should note that what is proven above is that for a circular orbit there are systematic variations with orbital phase over and above the significant random excursions. An eccentric orbit is a simple explanation for these systematic variations, but we cannot exclude other systematic effects. A cause for caution is that Barziv et al. (2001) found from their optical data an eccentricity which was larger than the value from the $\mathrm{X}$-ray pulsar in Vela X-1 (see their Fig. 18). Furthermore, model calculations of Zuiderwijk et al. (1977) showed that the radial-velocity curve for a tidally distorted star in a circular orbit might show an apparent eccentricity. The shape of the expected curve, however, is different from that observed. Given this, we cannot dismiss the possibility of a circular orbit out of hand, although we believe that the simplest and most likely solution is that it is in fact eccentric.

It is obvious that for some spectra the individual radialvelocity measurements deviate significantly from the best-fit 
Table 1. Journal of IUE SWP high-resolution spectra of HD 153919. Image numbers include an S or an L, refering to the small and large aperture, respectively. Phases are calculated from the ephemeris $T_{0}=\mathrm{JD} 2448900.873 \pm 0.015 ; P=3.411581 \pm 0.000027$ given by Rubin et al. (1996) including the orbital period change $\dot{P} / P=-(3.3 \pm 0.6) \times 10^{-6} \mathrm{yr}^{-1}$. The velocities are corrected for a shift of interstellar lines and are relative to that of SWP 1476 which was arbitrarily set to zero.

\begin{tabular}{|c|c|c|c|c|c|c|c|c|c|}
\hline \multicolumn{10}{|c|}{ Journal of observations } \\
\hline SWP & $\begin{array}{l}\text { JD }_{\text {mid.exp. }} \\
(-244000)\end{array}$ & Phase & $\begin{array}{r}v_{\text {rad }} \\
\left(\mathrm{km} \mathrm{s}^{-1}\right)\end{array}$ & $\begin{array}{l}1 \sigma \text {-error } \\
\left(\mathrm{km} \mathrm{s}^{-1}\right)\end{array}$ & SWP & $\begin{array}{l}\text { JD }_{\text {mid.exp. }} \\
(-244000)\end{array}$ & Phase & $\begin{array}{r}v_{\text {rad }} \\
\left(\mathrm{km} \mathrm{s}^{-1}\right)\end{array}$ & $\begin{array}{l}1 \sigma \text {-error } \\
\left(\mathrm{km} \mathrm{s}^{-1}\right)\end{array}$ \\
\hline $1476 \mathrm{~S}$ & 3632.610 & 0.8076 & 0.0 & 2.9 & $25586 \mathrm{~L}$ & 6160.579 & 0.7771 & -0.7 & 2.8 \\
\hline $1513 S$ & 3638.414 & 0.5087 & -3.2 & 5.9 & $25587 \mathrm{~L}$ & 6160.618 & 0.7885 & -5.7 & 2.9 \\
\hline $1714 S$ & 3664.042 & 0.0204 & 15.7 & 2.7 & $25588 \mathrm{~L}$ & 6160.665 & 0.8023 & 0.5 & 2.4 \\
\hline 1960L & 3700.269 & 0.6388 & -6.4 & 4.5 & $25589 \mathrm{~L}$ & 6160.715 & 0.8169 & 2.4 & 2.5 \\
\hline $1961 \mathrm{~L}$ & 3700.308 & 0.6502 & -6.5 & 4.3 & $25590 \mathrm{~L}$ & 6160.760 & 0.8300 & -0.4 & 2.6 \\
\hline 1969L & 3701.030 & 0.8618 & 1.9 & 3.4 & $25591 \mathrm{~L}$ & 6160.802 & 0.8424 & -1.1 & 2.7 \\
\hline 1970L & 3701.085 & 0.8779 & 1.9 & 2.7 & 25592L & 6160.844 & 0.8548 & 2.8 & 2.6 \\
\hline $1972 S$ & 3701.231 & 0.9207 & 5.5 & 3.8 & $25596 \mathrm{~L}$ & 6161.673 & 0.0977 & 21.1 & 2.4 \\
\hline $1973 S$ & 3701.280 & 0.9351 & 9.7 & 7.6 & $25597 \mathrm{~L}$ & 6161.706 & 0.1074 & 26.8 & 2.2 \\
\hline $1975 \mathrm{~S}$ & 3701.409 & 0.9729 & 4.6 & 2.9 & $25598 \mathrm{~L}$ & 6161.759 & 0.1229 & 22.6 & 2.6 \\
\hline $1982 \mathrm{~S}$ & 3702.224 & 0.2118 & 22.2 & 5.0 & $25599 \mathrm{~L}$ & 6161.808 & 0.1373 & 22.8 & 2.2 \\
\hline $1983 \mathrm{~S}$ & 3702.272 & 0.2258 & 23.6 & 3.3 & $25600 \mathrm{~L}$ & 6161.839 & 0.1463 & 25.8 & 2.2 \\
\hline $1986 \mathrm{~S}$ & 3702.404 & 0.2645 & 24.4 & 4.3 & $25607 \mathrm{~L}$ & 6162.582 & 0.3642 & 29.2 & 2.1 \\
\hline $1987 \mathrm{~S}$ & 3702.474 & 0.2851 & 36.0 & 2.1 & $25608 \mathrm{~L}$ & 6162.618 & 0.3748 & 29.3 & 2.3 \\
\hline $1991 \mathrm{~S}$ & 3703.099 & 0.4683 & 8.8 & 3.0 & 25609L & 6162.651 & 0.3844 & 25.6 & 2.2 \\
\hline $1992 \mathrm{~S}$ & 3703.148 & 0.4826 & 6.2 & 3.4 & $25610 \mathrm{~L}$ & 6162.689 & 0.3955 & 27.6 & 2.5 \\
\hline $1994 S$ & 3703.258 & 0.5148 & 5.4 & 2.9 & $25611 \mathrm{~L}$ & 6162.728 & 0.4070 & 25.5 & 2.3 \\
\hline $1995 \mathrm{~S}$ & 3703.308 & 0.5295 & 5.8 & 3.0 & $25612 \mathrm{~L}$ & 6162.764 & 0.4176 & 23.5 & 2.3 \\
\hline $2002 S$ & 3703.617 & 0.6200 & -4.7 & 2.1 & $25613 \mathrm{~L}$ & 6162.800 & 0.4280 & 21.8 & 2.2 \\
\hline $2003 S$ & 3703.708 & 0.6467 & -1.7 & 2.0 & $25614 \mathrm{~L}$ & 6162.833 & 0.4377 & 28.7 & 2.3 \\
\hline $2004 S$ & 3703.770 & 0.6649 & -0.7 & 2.1 & $25615 \mathrm{~L}$ & 6162.892 & 0.4551 & 17.0 & 2.1 \\
\hline $2006 S$ & 3704.067 & 0.7519 & -6.2 & 2.8 & $25616 \mathrm{~L}$ & 6162.929 & 0.4659 & 16.4 & 1.9 \\
\hline $2008 \mathrm{~S}$ & 3704.156 & 0.7780 & -12.9 & 3.0 & $25617 \mathrm{~L}$ & 6162.970 & 0.4780 & 12.0 & 1.8 \\
\hline $2009 S$ & 3704.206 & 0.7927 & -8.2 & 2.6 & $25618 \mathrm{~L}$ & 6163.005 & 0.4881 & 8.1 & 2.1 \\
\hline $2106 \mathrm{~S}$ & 3715.317 & 0.0494 & 17.6 & 2.5 & 25619L & 6163.044 & 0.4996 & 5.7 & 5.4 \\
\hline $2153 \mathrm{~S}$ & 3720.156 & 0.4677 & 9.2 & 2.3 & $25620 \mathrm{~L}$ & 6163.147 & 0.5298 & 0.1 & 2.3 \\
\hline $4742 S$ & 3957.201 & 0.9470 & 6.6 & 2.2 & $25621 \mathrm{~L}$ & 6163.187 & 0.5415 & -5.4 & 2.3 \\
\hline $4751 \mathrm{~S}$ & 3958.040 & 0.1929 & 34.6 & 2.2 & $28730 \mathrm{~L}$ & 6632.663 & 0.1509 & 31.3 & 2.5 \\
\hline $4752 \mathrm{~S}$ & 3958.088 & 0.2070 & 35.9 & 2.0 & $28731 \mathrm{~L}$ & 6632.707 & 0.1637 & 34.5 & 2.3 \\
\hline $4753 \mathrm{~S}$ & 3958.138 & 0.2216 & 36.4 & 2.5 & $28732 \mathrm{~L}$ & 6632.753 & 0.1772 & 34.3 & 2.4 \\
\hline $5180 \mathrm{~S}$ & 4003.047 & 0.3848 & 24.7 & 2.7 & & & & & \\
\hline
\end{tabular}

Table 2. Wavelength regions used for cross-correlation.

\begin{tabular}{ll}
\hline \hline Stellar & Interstellar \\
\hline $1305-1325$ & $1303.5-1305.5$ \\
$1337-1368$ & $1333.5-1335.5$ \\
$1425-1490$ & $1607.5-1609.5$ \\
$1755-1785$ & $1670.0-1672.0$ \\
$1810-1842$ & $1807.3-1808.7$ \\
\hline
\end{tabular}

Keplerian orbit. This was already known from the optical spectra. The Of supergiant has a strong stellar wind which can cause irregular variations in the radial velocities of some lines. Also the presence of a photo-ionization wake (Kaper et al. 1994) trailing the X-ray source may cause irregular small orbitto-orbit variations in the radial velocities. Nevertheless we devoted extra attention to some of the spectra. Particularly striking are the three velocities near phase 0.25 that are much lower than the other spectra at the same phase interval. These three are for spectra SWP1982, 1983 and 1986, which were taken in sequence. This would suggest an instrumental problem, were it not that SWP1987, which was taken immediately after SWP1986 at the same night as the mentioned three spectra, has a much higher velocity comparable with that of SWP 4751, 4752 and 4753. We compared the line profiles of all those spectra but could not find significant differences, although SWP 1982 and 1983 are very noisy. Note that the modest spectral resolution $(R \sim 10000)$ and low $S / N(\sim 20)$ does not allow for a detailed study of individual photospheric lines.

For HD 77581, the companion of Vela X-1, it was found that sequences of radial velocities showed deviations that were correlated over periods of about one day (Van Kerkwijk et al. 1995). To see whether such deviations are present in HD 153919 as well, in Fig. 1 lines connect residual velocities 


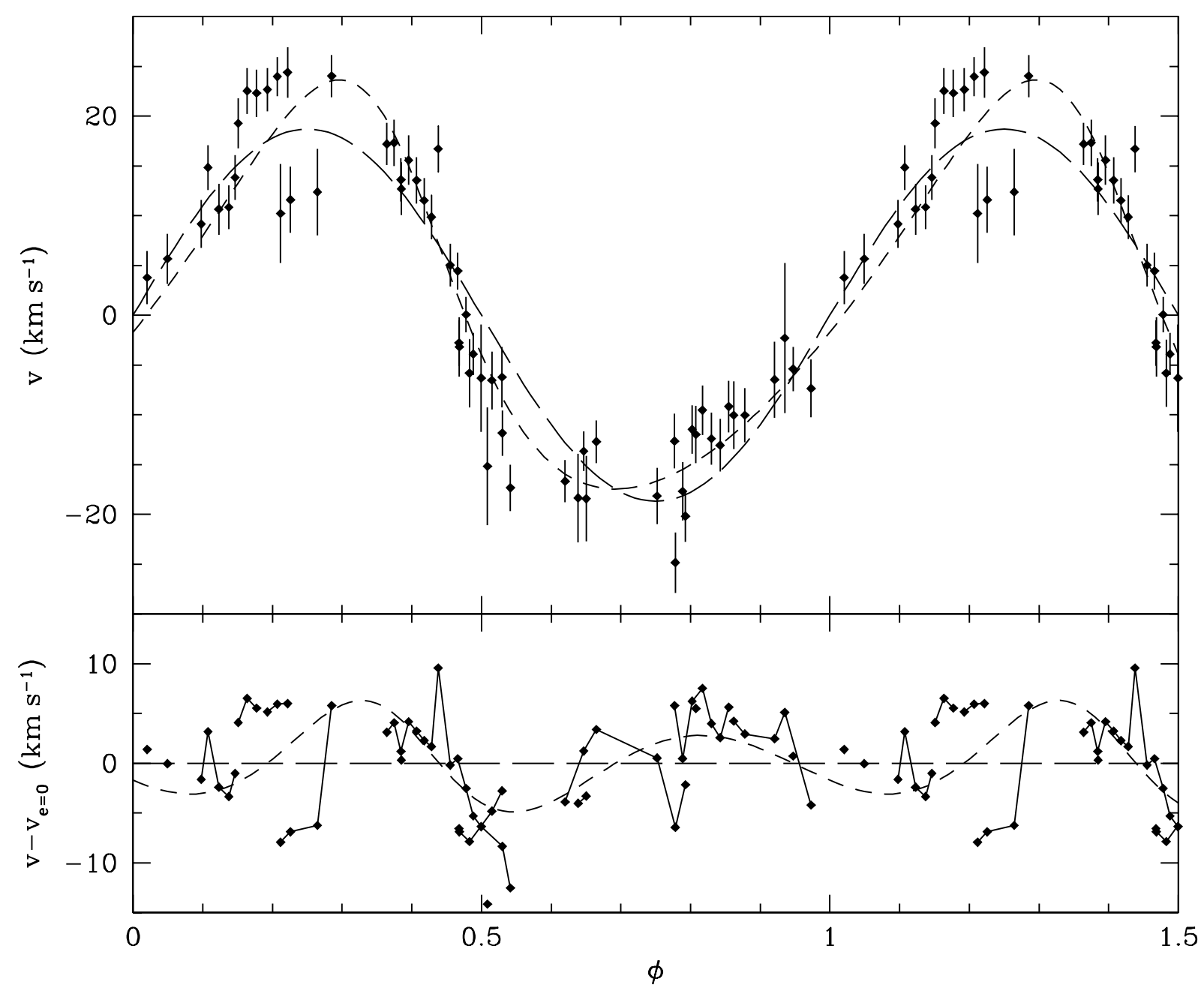

Fig. 1. Radial-velocity curve derived from the cross-correlated IUE spectra of HD 153919. The radial velocities in the top panel are plotted with error bars indicating the $1 \sigma$ uncertainties. Overdrawn is the Keplerian curve that best fits the data (short dashes) as well as the solution for a circular orbit (long dashes). The velocities have been shifted to zero system velocity based on the best fitting circular orbit (our analysis did not allow us to derive the systemic velocity; see text). In the lower panel, the residuals relative to the best-fit circular orbit are shown; the short-dashed curve shows where the residuals should lie for the best-fit eccentric orbit. For clarity, the error bars have been omitted. Points connected by lines were taken as a sequence.

for spectra that were taken as part of a sequence. Unfortunately, the result is inconclusive. Some groups seem to show correlated deviations, but for others the deviations seem random. It would be certainly worthwhile to monitor HD 153919 during some nights continuously to have a clue to the origin of those excursions and stellar wind variations that certainly influence and hamper an accurate determination of the orbital parameters.

Finally, we should mention that almost half of the set of spectra was taken in the small aperture of the SWP camera, the others in the large aperture (see Table 1). The spectra taken in the small aperture are the ones of the first years of IUE and have generally lower $S / N$. We determined orbital solutions for the small and large-aperture sets separately, but did not find significant differences.

\section{Comparison with earlier results}

From the present results we can conclude that the derived radial velocities are in good agreement with the results obtained much earlier from optical data (see Hammerschlag-Hensberge 1978).
Our results do not differ much from those by Heap \& Corcoran (1992) for the same data. The advantage of our analysis is that we use a larger part of the spectrum and correlate all spectra with each other, which, as a consequence, results in a smaller error on $K$. Remarkably, Heap \& Corcoran (1992) find a negligible eccentricity $(e \sim 0.003)$ with a very small error of the order of 0.003 . At first glance, the radial velocities in their Fig. 2 seem to fit equally well to an eccentric orbit. Also, from a statistical point of view, their errors on $e$ and $\omega$ are much too small. For small eccentricities the deviation from a circular orbit is a sinusoid with half the binary period and it is easy to see that $e \sim A(P / 2) / A(P)=A(P / 2) / K$, where $A$ is the amplitude of the sinusoid and $K$ the radial velocity amplitude. The error on $A(P / 2)$ will be comparable to the one on $K$ and as a consequence $\sigma_{e} \sim \sigma_{K} / K$. This implies $\sigma_{e} \sim 0.17$ for the error on the eccentricity, instead of $\sigma_{e}=0.003$ found by Heap \& Corcoran.

On the other hand, Stickland \& Lloyd (1993) find a stellar orbit with an eccentricity close to ours, but their results show a much smaller semi-amplitude $K$ of $10 \mathrm{~km} \mathrm{~s}^{-1}$. Contact by one of the authors with Dave Stickland for similar discrepant 
velocity values in the $\mathrm{X}$-ray binary Vela $\mathrm{X}$ - 1 has proven that the main cause for the discrepancy in the results is that their crosscorrelation procedure sets to zero the regions around interstellar lines and wind lines. When this is combined with slight inadequacies in normalization, artificial lines are introduced at fixed wavelengths in both spectra to be cross-correlated. As a consequence the radial velocities are biased systematically towards zero and the radial-velocity amplitude will be underestimated. A discrepancy of about $4 \mathrm{~km} \mathrm{~s}^{-1}$ had been found between the radial-velocity amplitude for the ultraviolet spectra of Vela X-1 analyzed by both groups (see Barziv et al. 2001 and Stickland et al. 1997). The reason for the much larger discrepancy of $10 \mathrm{~km} \mathrm{~s}^{-1}$ for HD 153919 might be the weaker and broader photospheric lines in this star combined with the better signal-to-noise ratio which give more striking errors when interstellar and wind lines are set to zero.

\section{Conclusions and evolutionary consequences}

The two main conclusions of this paper are that (i) the radialvelocity amplitude $K$ of HD 153919 determined from ultraviolet spectra is consistent with that derived from optical spectra; and (ii) the orbit most likely has a significant eccentricity. Note that the obtained value for $K$ depends on whether the orbit is eccentric $\left(K=20.6 \mathrm{~km} \mathrm{~s}^{-1}, e=0.22\right)$ or $\operatorname{not}\left(K=18.7 \mathrm{~km} \mathrm{~s}^{-1}\right)$.

Clark et al. (2002) performed a thorough study of the system parameters of HD 153919/4U 1700-37 and determined the most likely masses of both components using Monte Carlo simulations. These suggest a mass for HD 153919 and 4U 1700-37 of $M_{\star}=58 \pm 11 M_{\odot}$ and $M_{X}=2.4 \pm 0.3 M_{\odot}$, respectively. Clark et al. used the results described in this paper in their analysis, but prefered the orbital solution with zero eccentricity. As the mass function depends on the eccentricity:

$f=1.038 \times 10^{-7} K^{3} P\left(1-e^{2}\right)^{3 / 2}$

and

$\left(M_{\star}+M_{X}\right)^{2}=\frac{\left(M_{X} \sin i\right)^{3}}{f}$,

they noticed that the masses of both components become significantly higher when taking $e=0.22$. They argue that such high values for $M_{\star}$ are inconsistent with $\log g$ derived from the analysis of the photospheric spectrum. A non-zero eccentricity also results in a higher number of solutions that are rejected due to the inclination constraints. However, Clark et al. took the value of the radial-velocity amplitude belonging to the eccentric orbit $\left(K=20.6 \mathrm{~km} \mathrm{~s}^{-1}\right.$, so that $\left.f=0.0031 M_{\odot}\right)$, rather than $K=18.7 \mathrm{~km} \mathrm{~s}^{-1}$ for the circular orbit $\left(f=0.0023 M_{\odot}\right)$; for the latter value the masses would have become even higher. If one takes $K=20.6 \mathrm{~km} \mathrm{~s}^{-1}$ and $e=0.22$ then $f=0.0029 M_{\odot}$. Therefore, we conclude that the masses of both components are about $4 \%$ higher than proposed by Clark et al. (2002) if one uses the correct value for $f$ in case of the eccentric orbital solution.

The short orbital period of the system, combined with its eccentricity, will induce strong tidal interactions between the two components. Quaintrell et al. (2003) present evidence for tidally induced non-radial oscillations in HD 77581, the B-supergiant companion to Vela X-1, like 4U 1700-37 an eccentric $(e=0.09)$, wind-fed high-mass X-ray binary with an orbital period of 8.9 days. These non-radial oscillations may well be the cause of the radial-velocity excursions detected in Vela X-1. The radial-velocity excursions in HD 153919 could have a similar origin.

The eccentricity of the orbit of both Vela X-1 and 4U 170037 suggests that these systems have just entered the highmass X-ray binary phase and are on their way to become Roche-lobe overflow systems. The latter systems have circular orbits, possibly the result of tidal interaction. Both HD 77581 and HD 153919 do not rotate synchronously with their orbit: $P_{\text {orb }} / P_{\text {rot }}$ is 0.67 (Zuiderwijk 1995) and 0.46 (taking $v \sin i=$ $150 \mathrm{~km} \mathrm{~s}^{-1}, i=90^{\circ}, R_{\star}=21.9 R_{\odot}$ ), respectively, which may indicate that the supergiants are still expanding and that tidal forces have not (yet?) managed to synchronize the system and circularize the orbit.

Conti (1978) and Rappaport \& Joss (1983) suggested that the OB supergiants in HMXBs are too luminous for their mass. Kaper (2001) showed that the discrepancy between the measured and "spectroscopic" mass is most severe for the Rochelobe overflow systems, but is only modest for wind-fed systems such as Vela X-1. The proposed high mass of HD 153919 is consistent with its high luminosity, again suggesting that HD 153919 has not yet reached the phase of Roche-lobe overflow.

Acknowledgements. LK and MHvK acknowledge support from a fellowship of the Royal Netherlands Academy of Arts and Sciences.

\section{References}

Ankay, A., Kaper, L., De Bruijne, J. H. J., et al. 2001, A\&A, 370, 170 Barziv, O., Kaper, L., van Kerkwijk, M. H., Telting, J. H., \& van Paradijs, J. 2001, A\&A, 377, 925

Clark, J. S., Goodwin, S. P., Crowther, P. A., et al. 2002, A\&A, 392, 909

Conti, P. S. 1978, A\&A, 63, 255

Evans, N. R. 1988, IUE NASA Newsletter, 17, 53

Gies, D. R., \& Bolton, C. T. 1986, ApJS, 61, 419

Haberl, F., White, N. E., \& Kallman, T. R. 1989, ApJ, 343, 409

Hammerschlag-Hensberge, G. 1978, A\&A, 64, 399

Hammerschlag-Hensberge, G., Howarth, I. D., \& Kallman, T. R. 1990, ApJ, 352, 698

Heap, S. R., \& Corcoran, M. F. 1992, ApJ, 387, 340

Jones, C., Forman, W., Tananbaum, H., et al. 1973, ApJ, 181, L43

Kaper, L. 1998, in Boulder-Munich II: Properties of Hot, Luminous Stars, ed. Ian D. Howarth (San Francisco: Astron. Soc. of the Pacific), ASP Conf. Ser., 131, 427

Kaper, L. 2001, in The influence of binaries on stellar population studies, Astr. \& Space Science Lib., ed. D. Vanbeveren (Kluwer Acad. Publ.), 125

Kaper, L., Hammerschlag-Hensberge, G., \& Takens, R. J. 1990, Nature, 347, 652

Kaper, L., Hammerschlag-Hensberge, G., \& Zuiderwijk, E. J. 1994, A\&A, 289, 846

Humphreys, R. M. 1978, ApJS, 38, 309 
Quaintrell, H., Norton, A. J., Ash, T. D. C., et al. 2003, A\&A, 401, Stickland, D. J., Lloyd, C., \& Radzium-Woodham, A. 1997, MNRAS, 313 286, L21

Rappaport, S. A., \& Joss, P. C. 1983, in Accretion Driven Stellar X-ray Sources, ed. W. H. G. Lewin, \& E. P. J. van den Heuvel (Cambridge University Press), 1

Rubin, B. C., Finger, M. H., Harmon, B. A., et al. 1996, ApJ, 459, 259

Stickland, D. J. 1992, in Complementary Approaches to Double and Multiple Star Research, ed. McAlister, \& Hartkopf (Astron. Soc. of the Pacific, San Francisco), ASP Conf. Ser., 32, 393

Stickland, D. J., \& Lloyd, C. 1993, MNRAS, 264, 935

Van Kerkwijk, M. H. 1993, Ph.D. Thesis, University of Amsterdam Van Kerkwijk, M. H., Van Paradijs, J., \& Zuiderwijk, E. J. 1995a, A\&A, 303, 497

Van Kerkwijk, M. H., Van Paradijs, J., Zuiderwijk, E. J., et al. 1995b, A\&A, 303, 483

Van Paradijs, J., Takens, R., \& Zuiderwijk, E. J. 1977, A\&A, 57, 221

Zuiderwijk, E. J. 1995, A\&A, 299, 79 